\title{
Thermal Effect on Elastic Waves of Anisotropic Saturated Porous Solid
}

\author{
S. H. Guo \\ School of Civil Engineering and Architecture, Zhejiang University of Science and Technology, Hangzhou 310023, China \\ Correspondence should be addressed to S. H. Guo, gsh606@yahoo.com.cn \\ Received 6 March 2011; Accepted 25 October 2011 \\ Academic Editor: Ahmed Rachid \\ Copyright () 2011 S. H. Guo. This is an open access article distributed under the Creative Commons Attribution License, which \\ permits unrestricted use, distribution, and reproduction in any medium, provided the original work is properly cited. \\ The motion equations of anisotropic media, coupled to the mass conservation and thermoequilibrium equations of fluid, are \\ studied here based on the standard space of physical presentation for thermoelastic dynamics of anisotropic saturated porous \\ solids. By introducing a new compressible thermo-elastic model, a set of uncoupled equations of elastic waves are deduced. The \\ results show that the elastic waves and speeds of elastic waves are affected by both anisotropic subspaces of solids and thermal and \\ compressive coupling coefficients between fluid and solid. Based on these laws, we discuss the propagation behaviour of elastic \\ waves for various anisotropic solids.
}

\section{Introduction}

A general theory of three-dimensional propagation of elastic waves in a fluid-saturated porous solid was presented by Biot [1-3]. In this theory, the continuum consists of both solid constituent and fluid one, which are two entirely different materials, and the coupled, dilatational waves can be generated in both solid constituent and fluid one at a sound source. The thermal effects on the elastic waves were discussed by other works $[4,5]$. But in fact, for most of geological materials, such as rock and soil, it is difficult to distinguish the solid constituent and the fluid one and also impossible to get elastic waves of both solid constituent and fluid one by common measuring methods. In this paper, a new theory of elastic waves in a fluid-saturated porous solid subjected to thermal effects is given, in which the idea of standard spaces [6-13] is used to deal with the motion equation, thermoequilibrium equation, and the mass conservation equation. By this method, the classical Newton's equation of motion, thermoequilibrium equation, and the mass conservation equation under the geometric presentation can be transformed into the eigen ones under the physical presentation. The former is in the form of tensor, and the latter is in the form of scalar. By introducing a new compressible thermoelastic model, a set of uncoupled modal equations of elastic waves are obtained, each of which shows the existence of elastic subwaves; meanwhile, the propagation speed, propagation direction and space pattern of these subwaves can be completely determined by the modal equations.

\section{Constitutive Equations of Anisotropic Porous Media}

According to Terzaghi's effective stress theory and the compressible relationship of porous elastic materials, the constitutive equations of fluid saturated linear porous solids with thermal effects are as follows:

$$
\begin{gathered}
\sigma_{i j}=c_{i j k l}^{s} \varepsilon_{k l}^{s}+a_{i j} p-b_{i j} \theta, \\
n=\nu p+\kappa \theta-a_{k l} \varepsilon_{k l}^{s}, \\
\eta=\tau \theta+b_{k l} \varepsilon_{k l}^{s}+\kappa p
\end{gathered}
$$

where, $p$ is the pore pressure, $\theta$ is the temperature, $c_{i j k l}^{s}$ elastic tensor of solid constituent of porous material, $v$ compressive coefficient of porous material, $\tau$ heat coefficient, $a_{i j}$ coupling coefficient between solid constituent and fluid one, $b_{i j}$ thermal coupling coefficient, $\kappa$ coupling coefficient between porous deformation and heat, $n$ porosity, $\eta$ entropy. 
Rewriting (1) in the Voigt's notation, we have

$$
\begin{gathered}
\boldsymbol{\sigma}=\mathbf{c}^{s} \cdot \mathbf{S}^{s}+\mathbf{a} \cdot p-\mathbf{b} \theta, \\
n=\nu \cdot p+\kappa \theta-\mathbf{a}^{T} \cdot \mathbf{S}^{s}, \\
\eta=\tau \theta+\mathbf{b}^{T} \cdot \mathbf{S}^{s}+\kappa p .
\end{gathered}
$$

The skeleton elastic matrix $\mathbf{c}^{s}$ can be spectrally decomposed as follows [6-9]:

$$
\mathbf{c}^{\mathcal{s}}=\boldsymbol{\Phi} \Lambda \Phi^{T}
$$

where $\boldsymbol{\Lambda}=\operatorname{diag}\left[\lambda_{1}, \lambda_{2}, \lambda_{3}, \lambda_{4}, \lambda_{5}, \lambda_{6}\right]$ are the matrixes of eigen elasticity [10-13] of porous skeleton. $\Phi=$ $\left\{\varphi_{1}, \varphi_{2}, \varphi_{3}, \varphi_{4}, \varphi_{5}, \varphi_{6}\right\}$ are the modal matrixes, which are both orthogonal and positive definite matrixes, and satisfy $\boldsymbol{\Phi}^{T} \boldsymbol{\Phi}=\mathbf{I}$.

Projecting the elastic physical qualities of the geometric presentation, such as the stress vector $\boldsymbol{\sigma}$ and strain vector $\boldsymbol{S}^{s}$, into the standard spaces of the physical presentation, we get

$$
\begin{aligned}
& \boldsymbol{\sigma}^{*}=\boldsymbol{\Phi}^{T} \cdot \boldsymbol{\sigma}, \\
& \boldsymbol{S}^{*}=\boldsymbol{\Phi}^{T} \cdot \boldsymbol{S}^{s} .
\end{aligned}
$$

Rewriting (6) in the form of scalar, we have

$$
\begin{aligned}
\sigma_{i}^{*}=\boldsymbol{\varphi}_{i}^{* T} \cdot \boldsymbol{\sigma}, & i=1-m, \\
S_{i}^{*}=\boldsymbol{\varphi}_{i}^{* T} \cdot \mathbf{S}^{s}, & i=1-m,
\end{aligned}
$$

where $m(\leq 6)$ is the number of the elastic independent subspaces. Equations (7) and (8) show the elastic physical qualities under the physical presentation.

Substituting (6) into (2)-(4), respectively, and multiplying them with the transpose of modal matrix in the left, we have

$$
\begin{gathered}
\boldsymbol{\Phi}^{T} \boldsymbol{\sigma}=\boldsymbol{\Phi}^{T} \mathbf{c}^{s} \boldsymbol{\Phi} \mathbf{S}^{*}+\boldsymbol{\Phi}^{T} \mathbf{a} p-\boldsymbol{\Phi}^{T} \mathbf{b} \theta, \\
n=\nu \cdot p+\kappa \cdot \theta-\mathbf{a}^{T} \boldsymbol{\Phi} \mathbf{S}^{*}, \\
\eta=\tau \cdot \theta+\mathbf{b}^{T} \boldsymbol{\Phi} \mathbf{S}^{*}+\kappa \cdot p .
\end{gathered}
$$

Using (5), (6), we get

$$
\begin{aligned}
& \boldsymbol{\sigma}^{*}=\Lambda \mathbf{S}^{*}+\mathbf{a}^{*} p-\mathbf{b}^{*} \theta, \\
& n=\nu p+\kappa \theta-\mathbf{a}^{* T} \cdot \mathbf{S}^{*}, \\
& \eta=\tau \theta+\mathbf{b}^{* T} \cdot \mathbf{S}^{*}+\kappa p .
\end{aligned}
$$

Rewriting the above equations in the form of scalar, we have

$$
\begin{gathered}
\sigma_{i}^{*}=\lambda_{i} S_{i}^{*}+a_{i}^{*} p-b_{i}^{*} \theta, \quad i=1-m, \\
n=\nu p+\kappa \theta-a_{k}^{*} S_{k}^{*}, \quad k=1-m \text { sum to } k, \\
\eta=\tau \theta+b_{k}^{*} S_{k}^{*}+\kappa p, \quad k=1-m \text { sum to } k .
\end{gathered}
$$

Equations (11) are just the modal constitutive equations for anisotropic saturated linear porous elastic media with thermal effects.

\section{Mass Conservation, Heat, and Motion Equations}

The mass conservation equation of liquid in porous media is the following:

$$
\frac{\partial\left(n \gamma_{w}\right)}{\partial t}+\vec{\nabla} \cdot\left(\gamma_{w} \vec{V}\right)=0
$$

where, $n$ is porosity, $\gamma_{w}$ specific gravity of liquid, $\vec{V}$ flow velocity of liquid, and $\vec{\nabla}$ Hamilton operator.

For uncompressible and stable flowing of liquid, (12) can also be written as follows:

$$
\nabla_{t} n=0
$$

For thermoequilibrium state of an isolated system, the second law of thermodynamics is the following:

$$
d s=d s_{e}+d s_{i}=0,
$$

where $s$ is total entropy of system. By using the entropy density, the above can be written as follows

$$
\rho \dot{\eta}=0, \quad \text { or } \quad \rho \eta=C,
$$

where $C$ is an arbitrary constant, and we take it to be zero. The eigen equation of motion of solids can be written as follows [6-8]:

$$
\Delta_{i}^{*} \sigma_{i}^{*}=\rho_{s} \nabla_{t t} S_{i}^{*}, \quad i=1-m,
$$

where, $\Delta_{i}^{*}=\left\{\varphi_{i}^{*}\right\}^{T}[\Delta]\left\{\varphi_{i}^{*}\right\}$ is the stress operator, in which

$$
[\Delta]=\left[\begin{array}{cccccc}
\partial_{11} & 0 & 0 & 0 & \partial_{31} & \partial_{21} \\
0 & \partial_{22} & 0 & \partial_{32} & 0 & \partial_{21} \\
0 & 0 & \partial_{33} & \partial_{32} & \partial_{31} & 0 \\
0 & \partial_{23} & \partial_{23} & \left(\partial_{22}+\partial_{33}\right) & \partial_{21} & \partial_{31} \\
\partial_{13} & 0 & \partial_{13} & \partial_{12} & \left(\partial_{11}+\partial_{33}\right) & \partial_{32} \\
\partial_{12} & \partial_{12} & 0 & \partial_{13} & \partial_{23} & \left(\partial_{22}+\partial_{11}\right)
\end{array}\right],
$$

where $\partial_{i j}=\partial_{j i}=\partial^{2} / \partial x_{i} \partial x_{j}$.

\section{Modal Equation of Elastic Waves with Thermal Effects}

Substituting (11) into (13), (15), and (16), we have

$$
\begin{aligned}
\Delta_{i}^{*}\left(\lambda_{i} S_{i}^{*}+a_{i}^{*} p-b_{i}^{*} \theta\right) & =\rho_{s} \nabla_{t t} S_{i}^{*}, \\
\nu p+\kappa \theta-a_{k}^{*} S_{k}^{*} & =0, \\
\tau \theta+b_{k}^{*} S_{k}^{*}+\kappa p & =0 .
\end{aligned}
$$

From (19), we can get the following equation based on the operator principle:

$$
p=\frac{a_{k}^{*}}{\nu} S_{k}^{*}-\frac{\kappa}{\nu} \theta
$$


Substituting it into (18) and (20), respectively, we have

$$
\begin{aligned}
\Delta_{i}^{*}\left[\left(\lambda_{i}+\frac{a_{i}^{*} \delta_{i k} a_{k}^{*}}{v}\right) S_{i}^{*}-\left(\frac{\kappa}{v} a_{i}^{*}+b_{i}^{*}\right) \theta\right] & =\rho_{s} \nabla_{t t} S_{i}^{*}, \\
\left(\frac{\kappa \kappa}{v}-\tau\right) \theta-\left(b_{i}^{*}+\frac{\kappa}{v} a_{i}^{*}\right) S_{i}^{*} & =0 .
\end{aligned}
$$

Substituting (23) into (22) again, we get

$$
\begin{aligned}
\Delta_{i}^{*}[ & \left(\lambda_{i}+\frac{a_{i}^{*} \delta_{i k} a_{k}^{*}}{v}\right)-\left(\frac{\kappa}{v} a_{i}^{*}+b_{i}^{*}\right) \\
& \left.\times\left(\frac{\kappa \kappa}{v}-\tau\right)^{-1}\left(b_{i}^{*}+\frac{\kappa}{v} a_{i}^{*}\right)\right] S_{i}^{*}=\rho_{s} \nabla_{t t} S_{i}^{*} .
\end{aligned}
$$

Rewriting it in the standard form of elastic waves, we have

$$
\Delta_{i}^{*} S_{i}^{*}=\frac{1}{c_{i}^{2}} \nabla_{t t} S_{i}^{*}, \quad i=1-m
$$

where

$$
c_{i}=\sqrt{\frac{\left[\left(\lambda_{i}+\left(a_{i}^{*} \delta_{i k} a_{k}^{*} / \nu\right)\right)-\left((\kappa / \nu) a_{i}^{*}+b_{i}^{*}\right)^{2}((\kappa \kappa / \nu)-\tau)^{-1}\right]}{\rho_{s}}} .
$$

It is just the speed of elastic waves in porous media when thermal effects are considered.

\section{Application}

5.1. Isotropic Media. For isotropic media, the material tensors in (1) are represented by the following matrices under the compact notation

$$
\left[\begin{array}{cccccc}
c_{11} & c_{12} & c_{12} & 0 & 0 & 0 \\
c_{12} & c_{11} & c_{12} & 0 & 0 & 0 \\
c_{12} & c_{12} & c_{11} & 0 & 0 & 0 \\
0 & 0 & 0 & c_{66} & 0 & 0 \\
0 & 0 & & & c_{66} & 0 \\
0 & 0 & 0 & 0 & 0 & c_{66}
\end{array}\right],\left[\begin{array}{ccc}
a_{11} & 0 & 0 \\
0 & a_{11} & 0 \\
0 & 0 & a_{11}
\end{array}\right],\left[\begin{array}{ccc}
b_{11} & 0 & 0 \\
0 & b_{11} & 0 \\
0 & 0 & b_{11}
\end{array}\right],
$$

where $c_{66}=\left(c_{11}-c_{12}\right)$.

There are two independent eigenspaces in an isotropic solids [6-9]

$$
W=W_{1}^{(1)}\left[\boldsymbol{\varphi}_{1}\right] \oplus W_{2}^{(5)}\left[\boldsymbol{\varphi}_{2}, \ldots, \boldsymbol{\varphi}_{6}\right],
$$

where

$$
\begin{gathered}
\varphi_{1}=\frac{\sqrt{3}}{3}[1,1,1,0,0,0]^{T}, \quad \varphi_{2}=\frac{\sqrt{2}}{2}[0,1,-1,0,0,0]^{T} \\
\varphi_{3}=\frac{\sqrt{6}}{6}[2,-1,-1,0,0,0]^{T}, \quad \varphi_{i}=\xi_{i} \quad(i=4,5,6)
\end{gathered}
$$

and $\xi_{i}$ is a vector of order 6 in which the $i$ th element is 1 and others are 0 .

The eigenelasticity of isotropic solids are

$$
\begin{gathered}
\lambda_{1}=c_{11}+2 c_{12}=3 \lambda+2 \mu, \\
\lambda_{2}=c_{11}-c_{12}=2 \mu,
\end{gathered}
$$

where $\lambda$ and $\mu$ are Lame's constants.

The coupled coefficients and stress operators for isotropic solids are calculated as

$$
\begin{array}{cc}
a_{1}^{*}=\sqrt{3} a_{11}, & a_{2}^{*}=0, \\
b_{1}^{*}=\sqrt{3} b_{11}, & b_{2}^{*}=0, \\
\Delta_{1}^{*}=\frac{1}{3} \nabla_{\mathrm{III}}^{2}, & \Delta_{2}^{*}=\frac{1}{2} \nabla_{\mathrm{III}}^{2},
\end{array}
$$

where $\nabla_{\text {III }}^{2}$ is Laplace's operator of three dimensions.

It will be seen as follows that the modal strains in isotropic case represent the dilation and shear deformation, respectively. is

Using (8), the modal strain of order 1 of isotropic solids

$$
S_{1}^{*}=\varphi_{1}^{* T} \cdot \mathbf{S}=\frac{\sqrt{3}}{3}\left(S_{11}+S_{22}+S_{33}\right)
$$

Equation (32) represents the relative change of the volume of elastic solids. So the wave equation of order 1 shows the motion of pure longitudinal wave.

Also from (8), the modal strain of order 2 of isotropic solids is

$$
S_{2}^{*} \varphi_{2}^{*}=\mathrm{S}-S_{1}^{*} \varphi_{1}^{*}
$$

Using the orthogonality condition of eigenvectors, we have

$$
\begin{aligned}
\left|S_{2}^{*}\right| & =\left[\left(\mathbf{S}-S_{1}^{*} \boldsymbol{\varphi}_{1}^{*}\right)^{T} \cdot\left(\mathbf{S}-S_{1}^{*} \boldsymbol{\varphi}_{1}^{*}\right)\right]^{1 / 2} \\
& =\left\{\frac{1}{3}\left[\left(S_{1}-S_{2}\right)^{2}+\left(S_{2}-S_{3}\right)^{2}+\left(S_{3}-S_{1}\right)^{2}\right]\right\}^{1 / 2} .
\end{aligned}
$$

Equation (34) represents the pure shear strain on the elastic solids. So the wave equation of order 2 shows the motion of pure transverse wave as follows:

$$
\begin{gathered}
c_{1}=\sqrt{\frac{\left[\left(K+\left(a_{11}^{2} / \nu\right)\right)-\left((\kappa / \nu) a_{11}+b_{11}\right)^{2}((\kappa \kappa / \nu)-\tau)^{-1}\right]}{\rho_{s}}}, \\
c_{2}=\sqrt{\frac{G}{\rho_{s}}},
\end{gathered}
$$

where $K$ is the bulk modulus and $G$ shear one of solid. It is seen that the speed of pure transverse wave is not subject to the thermal effects. 
5.2. Transversely Isotropic Media. For isotropic media, the material tensors in (1) are represented by the following matrices under the compact notation:

$$
\left[\begin{array}{cccccc}
c_{11} & c_{12} & c_{13} & 0 & 0 & 0 \\
c_{12} & c_{11} & c_{13} & 0 & 0 & 0 \\
c_{13} & c_{13} & c_{13} & 0 & 0 & 0 \\
0 & 0 & 0 & c_{44} & 0 & 0 \\
0 & 0 & & & c_{44} & 0 \\
0 & 0 & 0 & 0 & 0 & c_{66}
\end{array}\right],\left[\begin{array}{ccc}
a_{11} & 0 & 0 \\
0 & a_{11} & 0 \\
0 & 0 & a_{33}
\end{array}\right],\left[\begin{array}{ccc}
b_{11} & 0 & 0 \\
0 & b_{11} & 0 \\
0 & 0 & b_{33}
\end{array}\right],
$$

where $c_{66}=(1 / 2)\left(c_{11}-c_{12}\right)$.

There are four independent eigenspaces in an transversely isotropic solids [6-9] as follows:

$W=W_{1}^{(1)}\left[\boldsymbol{\varphi}_{1}\right] \oplus W_{2}^{(1)}\left[\boldsymbol{\varphi}_{2}\right] \oplus W_{3}^{(2)}\left[\boldsymbol{\varphi}_{3}, \boldsymbol{\varphi}_{6}\right] \oplus W_{4}^{(2)}\left[\boldsymbol{\varphi}_{4}, \boldsymbol{\varphi}_{5}\right]$

where

$$
\begin{aligned}
\boldsymbol{\varphi}_{1,2}= & \frac{c_{13}}{\sqrt{\left(\lambda_{1,2}-c_{11}-c_{12}\right)^{2}+2 c_{13}{ }^{2}}} \\
& \times\left[1,1, \frac{\lambda_{1,2}-c_{11}-c_{12}}{c_{3}}, 0,0,0\right]^{T} \\
\varphi_{3}= & \frac{\sqrt{2}}{2}[1,-1,0,0,0,0]^{T}, \quad \varphi_{\mathbf{i}}=\xi_{i}, \quad i=4,5,6 .
\end{aligned}
$$

The eigenelasticities of transversely isotropic solids are

$$
\begin{gathered}
\lambda_{1,2}=\frac{c_{11}+c_{12}+c_{33}}{2} \pm \sqrt{\left(\frac{c_{11}+c_{12}+c_{33}}{2}\right)^{2}+2 c_{13}{ }^{2}} \\
\lambda_{3}=c_{11}-c_{12}, \quad \lambda_{4}=c_{44} .
\end{gathered}
$$

The coupled coefficients and stress operators for transversely isotropic solids are calculated as

$$
\begin{gathered}
a_{i}^{*}=g_{i}\left(2 a_{11}+d_{i} a_{33}\right), \quad i=1,2, \\
a_{3}^{*}=0, \quad a_{4}^{*}=0, \\
b_{i}^{*}=g_{i}\left(2 b_{11}+d_{i} b_{33}\right), \quad i=1,2, \\
b_{3}^{*}=0, \quad b_{4}^{*}=0, \\
\Delta_{1,2}^{*}=g_{1,2}^{2} \nabla_{\mathrm{III}}^{*}, \quad \Delta_{3}^{*}=\frac{2}{3} \nabla_{\mathrm{II}}^{2}, \quad \Delta_{4}^{*}=\frac{1}{2}\left(\nabla_{\mathrm{III}}^{2}+2 \partial_{12}\right),
\end{gathered}
$$

where, $g_{i}=c_{13} / \sqrt{\left(\lambda_{i}-c_{11}-c_{12}\right)^{2}+2 c_{13}{ }^{2}}, d_{i}=\lambda_{i}-c_{11}-$ $c_{12} / c_{3}$.
It will be seen as follows that the modal strains in transversely isotropic case represent the quasi-dilation and quasishear deformation, respectively:

$$
\begin{aligned}
S_{1,2}{ }^{*}= & \frac{c_{13}}{\sqrt{\left(\lambda_{1,2}-c_{11}-c_{12}\right)^{2}+2 c_{13}^{2}}} \\
& \times\left[S_{11}+S_{22}+\left(\frac{\lambda_{1,2}-c_{11}-c_{12}}{c_{13}}\right) S_{33}\right], \\
S_{3}{ }^{*}= & \sqrt{\frac{1}{2}\left(S_{11}+S_{22}\right)^{2}+S_{12}^{2}}, \\
S_{4}{ }^{*}= & \sqrt{\frac{1}{2}\left(S_{32}^{2}+S_{31}^{2}\right) .}
\end{aligned}
$$

The speeds of transversely isotropic solids are the following:

$c_{1,2}=\sqrt{\frac{\left[\left(\lambda_{1,2}+\left(a_{1,2}^{* 2} / \nu\right)\right)-\left((\kappa / \nu) a_{1,2}^{*}+b_{1,2}^{*}\right)^{2}((\kappa \kappa / \nu)-\tau)^{-1}\right]}{\rho_{s}}}$,

$c_{3}=\sqrt{\frac{c_{11}-c_{12}}{\rho_{s}}}$,

$c_{4}=\sqrt{\frac{c_{44}}{\rho_{s}}}$.

It is seen that the speeds of quasi-transverse waves are not subject to the thermal effects.

\section{Conclusion}

In this paper, a new elastic wave model for fluid-saturated porous solid with thermal effects under single-phase assumption is presented, in which the mechanical equations of motion coupled to mass conservation equation and thermoequilibrium equation are considered. A set of uncoupled elastic wave equations for anisotropic porous solid with thermal effects are deduced. The results show that the thermal and compressive coupling coefficients between solid and liquid constituents of system has and shear deformation of solids have not effects on the elastic wave equations and propagation speeds of elastic waves for anisotropic porous solids.

\section{References}

[1] M. A. Biot, "Theory of propagation of elastic waves in a fluidsaturated porous solid: I. Low-frequency range," Journal of the Acoustical Society of America, vol. 28, no. 2, pp. 168-178, 1956.

[2] M. A. Biot, "Theory of propagation of elastic waves in a fluidsaturated porous solid: II. Higher frequency range," Journal of the Acoustical Society of America, vol. 28, no. 2, pp. 179-191, 1956.

[3] M. A. Biot, "Mechanics of deformation and acoustic propagation in porous media," Journal of Applied Physics, vol. 33, no. 4, pp. 1482-1498, 1962.

[4] C. Pecker and H. Deresiewicz, "Thermal effects on wave propagation in liquid-filled porous media," Acta Mechanica, vol. 16, no. 1-2, pp. 45-64, 1973. 
[5] V. De La Cruz and T. J. T. Spanos, "Thermomechanical coupling during seismic wave propagation in a porous medium," Journal of Geophysical Research, vol. 94, no. 1, pp. 637-642, 1989.

[6] S. Guo, "An eigen theory of rheology for complex media," Acta Mechanica, vol. 198, no. 3-4, pp. 253-260, 2008.

[7] S. H. Guo, "Eigen theory of viscoelastic dynamics based on the Kelvin-Voigt model," Applied Mathematics and Mechanics, vol. 25, no. 7, pp. 792-798, 2004.

[8] S. H. Guo, "An eigen theory of electromagnetic waves based on the standard spaces," International Journal of Engineering Science, vol. 47, no. 3, pp. 405-412, 2009.

[9] S. Guo, "An eigen theory of waves in piezoelectric solids," Acta Mechanica Sinica, vol. 26, no. 2, pp. 241-246, 2010.

[10] W. Thomson, "Elements of a mathematical theory of elasticity," Philosophical Transactions of the Royal Society of London, vol. 146, pp. 481-498, 1856.

[11] M. M. Mehrabadi and S. C. Cowin, "Eigentensors of linear anisotropic elastic materials," Quarterly Journal of Mechanics and Applied Mathematics, vol. 43, no. 1, pp. 15-41, 1990.

[12] K. Helbig, Foundations of Anisotropy for Exploration Seismics, Handbook of Geophysical Exploration: Seismic Exploration, Pergamon Press, 1994.

[13] J. M. Carcione and F. Cavallini, "A rheological model for anelastic anisotropic media with applications to seismic wave propagation," Geophysical Journal International, vol. 119, no. 1, pp. 338-348, 1994. 

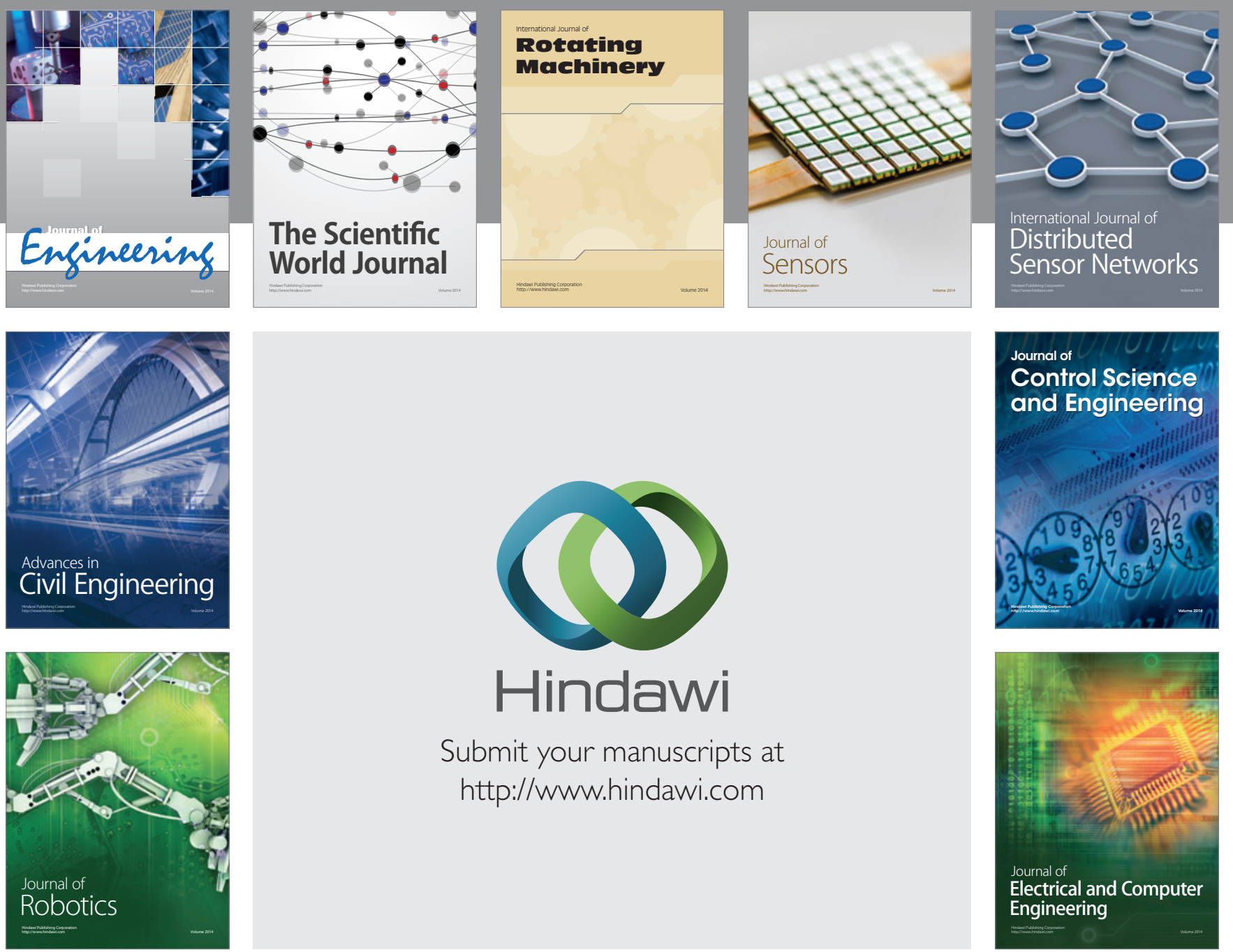

Submit your manuscripts at

http://www.hindawi.com
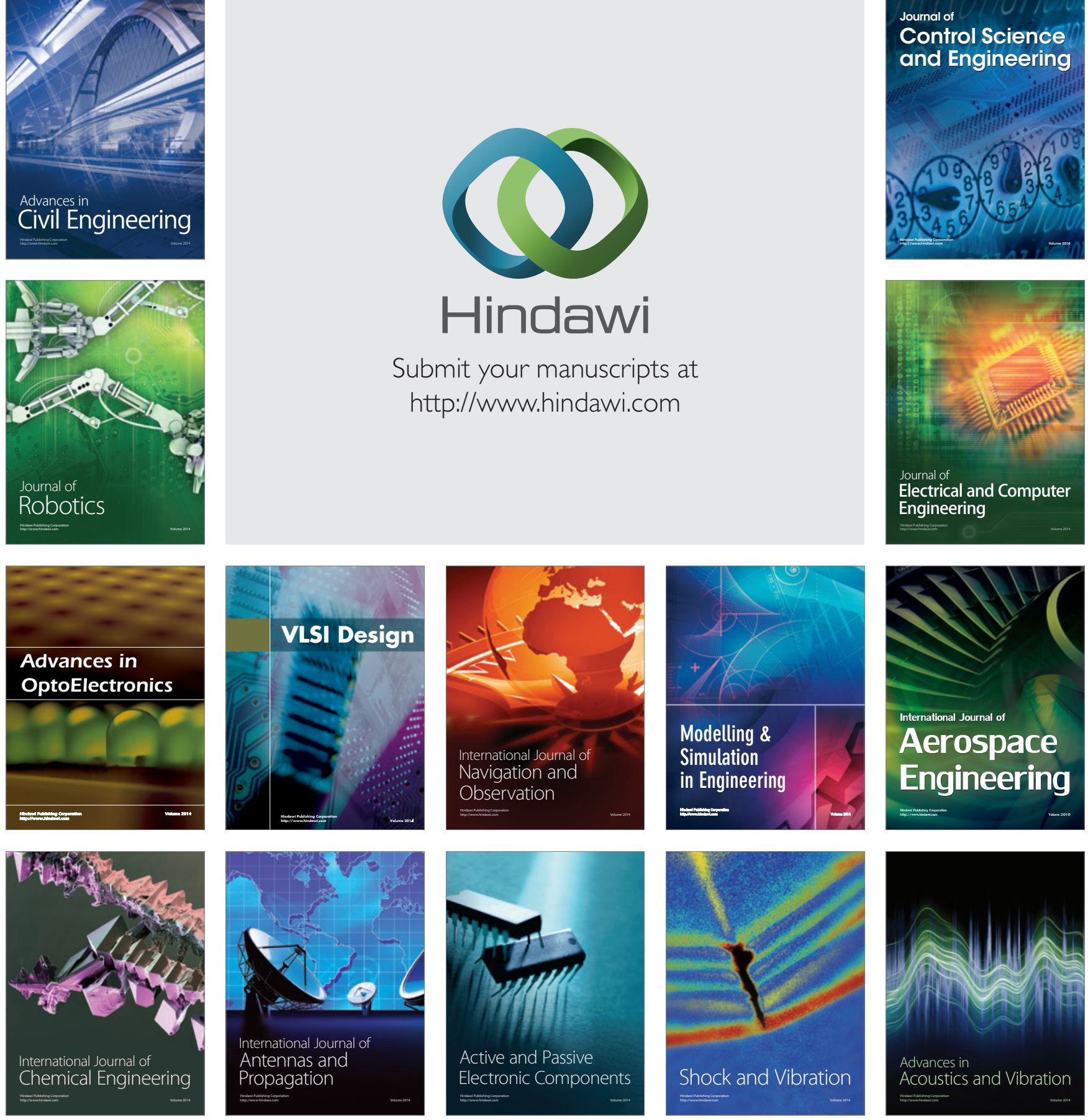